Hiroki Matsuura, MD

Department of General Internal

Medicine, Okayama City Hospital,

Okayama, Japan
Yu Suganami, MD, PhD

Department of General Internal

Medicine, Okayama City Hospital,

Okayama, Japan

\title{
Hypothyroidism, eyelash loss
}

\begin{abstract}
A 77-YEAR-OLD WOMAN presented to our outpatient department with chronic general fatigue and peripheral edema. She had mild hypertension and diabetes but no significant cardiovascular or gastrointestinal problems.

Physical examination revealed nonpitting edema in the legs and loss of eyelashes (Figure 1) from all four eyelids. Her eyebrows were also thin, but she said she usually trims them for makeup. She denied rubbing the eyelid, pulling the eyelashes, or having experienced previous eyelid injuries.

Laboratory testing showed a high thyroidstimulating hormone level of $33.7 \mu \mathrm{U} / \mathrm{mL}$ (reference range 0.35-4.94), a low serum thyroxine level, and the presence of antithyroid antibodies.

Based on the presentation and findings, the diagnosis was hypothyroidism with milphosis.
\end{abstract}

\section{CAUSES OF MILPHOSIS}

Terms used for hair loss include milphosis, madarosis, and alopecia. Milphosis is loss of eyelashes, madarosis is loss of eyelashes and eyebrow hairs, and alopecia is general loss of hair, but especially from the head.

Eyelashes and eyebrows have many roles such as protecting the eye (by preventing sweat and water from entering), cosmetic appearance, and social communication.

Typically, milphosis is caused by thyroid disorders, as in this case. Both insufficient and excessive levels of thyroid hormones are associated with hair growth and hair follicle cycling. In particular, hypothyroidism can cause telogen effluvium (thinning or shedding of hair resulting from the early entry of the hair follicle into the resting phase), early graying, and reduced tensile strength.

The authors report no relevant financial relationships which, in the context of their contributions, could be perceived as a potential conflict of interest. doi:10.3949/ccjm.87a.20009

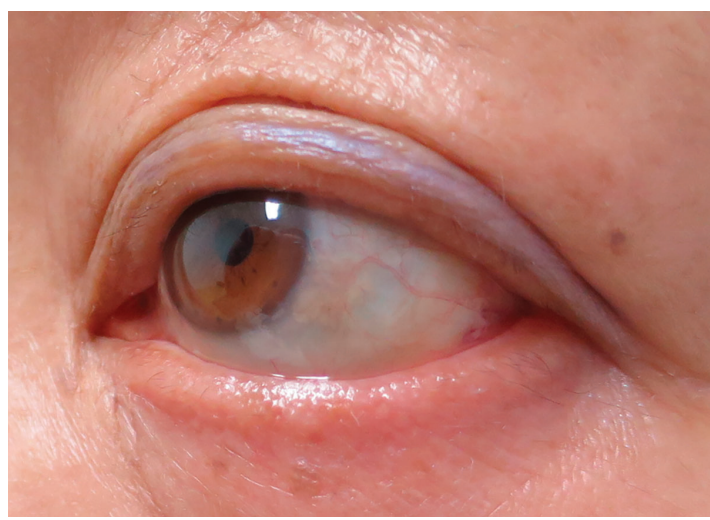

Figure 1.

Milphosis is also associated with a range of diseases from localized dermatologic disease to systemic disorders such as hypoparathyroidism, infection (herpes zoster, tuberculosis, syphilis, trachoma, Hansen disease), radiation, drug effects (heparin, androgens, retinoids, and angiotensin-converting enzyme inhibitors), injury, toxins (cocaine and thallium), zinc deficiency, biotin deficiency, psoriasis, systemic lupus erythematosus, discoid lupus, and neoplasm (basal cell carcinoma and squamous-cell carcinoma). ${ }^{1}$

Milphosis can sometimes be useful as a physical symptom when it helps detect underlying systemic disorders. However, clinicians should also consider trichotillomania in the differential diagnosis of patients with milphosis whose clinical history and laboratory findings are unclear. In nonscarring milphosis, hair can regrow after the primary disease is appropriately treated.

Our patient received supplemental thyroxine, but her eyelashes did not grow back.

\section{REFERENCES}

1. Jordan DR. Eyelash loss. Semin Plast Surg 2007; 21(1):3236. doi:10.1055/s-2007-967745

Address: Hiroki Matsuura, MD, Department of General Internal Medicine, Okayama City Hospital, 3-20-1, Omote-cho, Kitanagase, Okayama-city, Okayama, 700-0962, Japan; superonewex0506@yahoo.co.jp
Testing showed a high thyroidstimulating hormone level, low serum thyroxine, and presence of antithyroid antibodies 